\title{
31
}

\section{Electric Current Wound Healing}

\author{
David Cukjati
}

Laboratory of Biocybernetics, Faculty of Electrical Engineering, Ljubljana, Slovenia

\section{Rajmond Šavrin}

Institute for Rehabilitation, Republic of Slovenia

Ljubljana, Slovenia

The authors review the existing physical modalities for treatment of chronic wounds and show the advantages of electric current and electromagnetic field stimulation. Direct currents, low frequency pulsed currents, monophasic high voltage pulses and pulsed electromagnetic fields are compared in respect of their efficiency. Wound healing quantification methods, wound healing dynamics and prognostic factors in the prediction of wound healing proposed by the authors represent significant contribution in understanding the mechanisms of electric wound healing.

\section{WOUND HEALING}

Skin is a vital organ, in the sense that the loss of substantial fraction of its mass immediately threatens the life of the individual. A cutaneous wound is any loss of skin integrity. Such a loss can result suddenly, either from fire or mechanical accident, or it can occur in a chronic manner due to illness, as in skin ulcers. Since intact skin is of vital importance to protect the organism against environment, regenerative mechanisms must be activated to resolve a defect. Cutaneous wound healing is a dynamic biological process that begins with tissue injury. It has several goals:

The discontinuation of further injury

The recruitment of injured cells

The formation of new tissue

The remodeling of the new tissue to best approximate the preinjury form and function.

These events have traditionally been divided into three overlapping phases: an inflammatory phase, a proliferative phase, and a maturation phase. 
The inflammatory phase refers to immediate vascular and inflammatory response to injury. The immediate response to blood vessel disruption is activation of the coagulation cascade and the production of blood clot. After several minutes, an acute inflammatory response ensues. Subsequently, leukocytes clear the wound of debris and release growth factors to initiate the healing process. Then follows the proliferative phase involving deposition and formation of granulation tissue, which becomes a new and temporary weak tissue, reepitelization, and wound shrinkage. The maturation phase is characterized clinically by gradual shrinking, thinning, and paling of the scar, leading to decreased bulk but increased tensile strength (1). If any of three overlapping phases of cutaneous wound healing is suppressed, wound healing is prolonged or even prevented. Reasons for slower or retarded healing can be local, such as bacterial infection that prolongs the inflammatory phase; lower oxygen tension that prolongs the proliferative phase; or systemic, such as injuries of the nervous system, diabetes mellitus, atherosclerosis and other vascular diseases, metabolic and ageing problems that affect one or more phases of wound healing.

When conservative methods of wound care cannot facilitate wound healing, the wound is considered to be chronic. Such chronic wounds can last for weeks, months, or even years despite adequate and appropriate care. They are difficult and frustrating to manage. Typical chronic wounds are pressure ulcers in spinal-cord-injured patients, ischemic ulcers in lower extremities of patients with peripheral vascular disease, ulcers in geriatric patients, and wounds after limb amputations (2). Patients are subjected to discomfort, stress, and high cost of long-term conventional treatment required for such ulceration to heal.

According to statistic reports, $11 \%$ of all hospitalized patients and up to $20 \%$ of all elderly home residents suffer from decubital wounds (3). The frequency of decubital wounds in spinal-cord-injured (SCI) patients is quite diverse, ranging from $23 \%$ to as much as $85 \%$ / year (4). The percentage of SCI patients that will end up with at least one decubital wound in their lifetime is $85 \%(5-7)$. Decubital wounds may be directly responsible for death of $7-8 \%$ of patients, while frequently an indirect influence upon the mortality rate through different health complications, such as osteomyelitis or sepsis (8). Diabetic foot ulcers are a common problem and result in more than 85,000 lower extremity amputations each year in the United States. Studies were employed to find the most cost effective treatment of nonhealing wounds (9).

Chronic wound healing represent a major social, medical and economic problem. Therefore an extensive effort has been done to find any treatment modality, which may accelerate the wound-healing process.

\section{TREATMENT OF CHRONIC WOUNDS}

The understanding of the biological and pathologic events in wound healing has led to three areas of treatment that are currently indicated for the treatment of chronic wounds in the clinic practice (10): grow factors, tissue engineered skin, and physical devices. Despite the vast interest in growth factors and cytokine biology and their potential for wound healing $(11,12)$, clinical trials to accelerate chronic wound healing have in most cases been disappointing. Nevertheless several studies have shown that the application of growth factors may induce the acceleration of cutaneous wound healing in animal models (13). Tissue engineered skin offers the possibility of creating physiologically compatible human skin and are successfully used on burn wounds to prevent bacterial infection and allow the wound the chance to heal by normal reparative processes. Unlike in burn patients, the condition in patients with chronic wounds results from underlying diseases therefore closing wound with skin substitutes would not be sufficient to initiate the wound healing (14). A 
review of the literature revealed that many adjunctive physical devices were employed and reported to facilitate chronic wound healing, including wound dressings (15), low-level laser therapy (16), low-intensity laser therapy-combined phototherapy (17), ultrasound (18), ultrasound/ultraviolet treatment (19), hyperbaric oxygen (20), electric current stimulation $(21,22)$ and magnetic-electromagnetic field stimulation (23).

According to Sheffet et al. (24) only two treatment-related recommendations receive high ratings for reported experimental evidence of validity: use of moist wound dressings and adjunctive electrical stimulation for nonhealing wounds. The recent reviews of literature revealed advances in the knowledge of electrical wound healing.

\section{ELECTRIC CURRENT AND ELECTROMAGNETIC FIELD STIMULATION}

Electrical interactions are regulators of many basic physiological processes ranging from conformation of molecules within a cell membrane bilayer to the macroscopic mechanical properties of the tissues. However, there is no well-established mechanism that can explain how weak electric currents and electromagnetic field (EMF) applications affect the behavior of living cells and tissues. The use of electric current and EMF stimulation to enhance wound healing is not new. The pioneer clinical studies range in late 1960s (25). In recent years, electric current and EMF stimulation (electrical stimulation) have become increasingly popular treatment modalities of nonhealing wounds. Electrical stimulation was primarily used to accelerate healing of decubitus ulcers and vein insufficiency. Studies revealed that pressure sores react better on electrical stimulation than other types of wounds (26). In the literature following positive effects of electrical stimulation on chronic wound healing can be found:

Accelerated epitelization and healing

Higher percentage of healed wounds in comparing to conservative treatment and activation of healing when conventional treatment failed

Prevention of tissue necrosis and antibacterial effect

Improved blood circulation

Increased wound contraction

Higher scar elasticity

Increased response of fibroblasts

Decrease of neuropath pain

Decreased peripheral neuropathy

There have been many excellent reviews published on electrical wound healing $(21,22,24,27)$. The results of the first meta-analysis showed that electrical wound healing is effective adjective therapy for chronic wound healing, while relative effectiveness of different types of electrical wound healing is inconclusive (28). Nevertheless, more recent meta-analysis on selected pooled trials could not constitute acceptable proof that electrical stimulation has specific effect on health (29). The survey of existing literature indicates a variety of electric and electromagnetic modalities that have been developed to heal wounds (Table 1) (30-35). Only little uniformity can be found in the literature reporting the use of electrical stimulation with respect to electrical signal properties, placement of stimulation electrodes, and treatment regime. Although electrical stimulation produces a substantial improvement in the healing of chronic wounds, further research is needed to identify which 


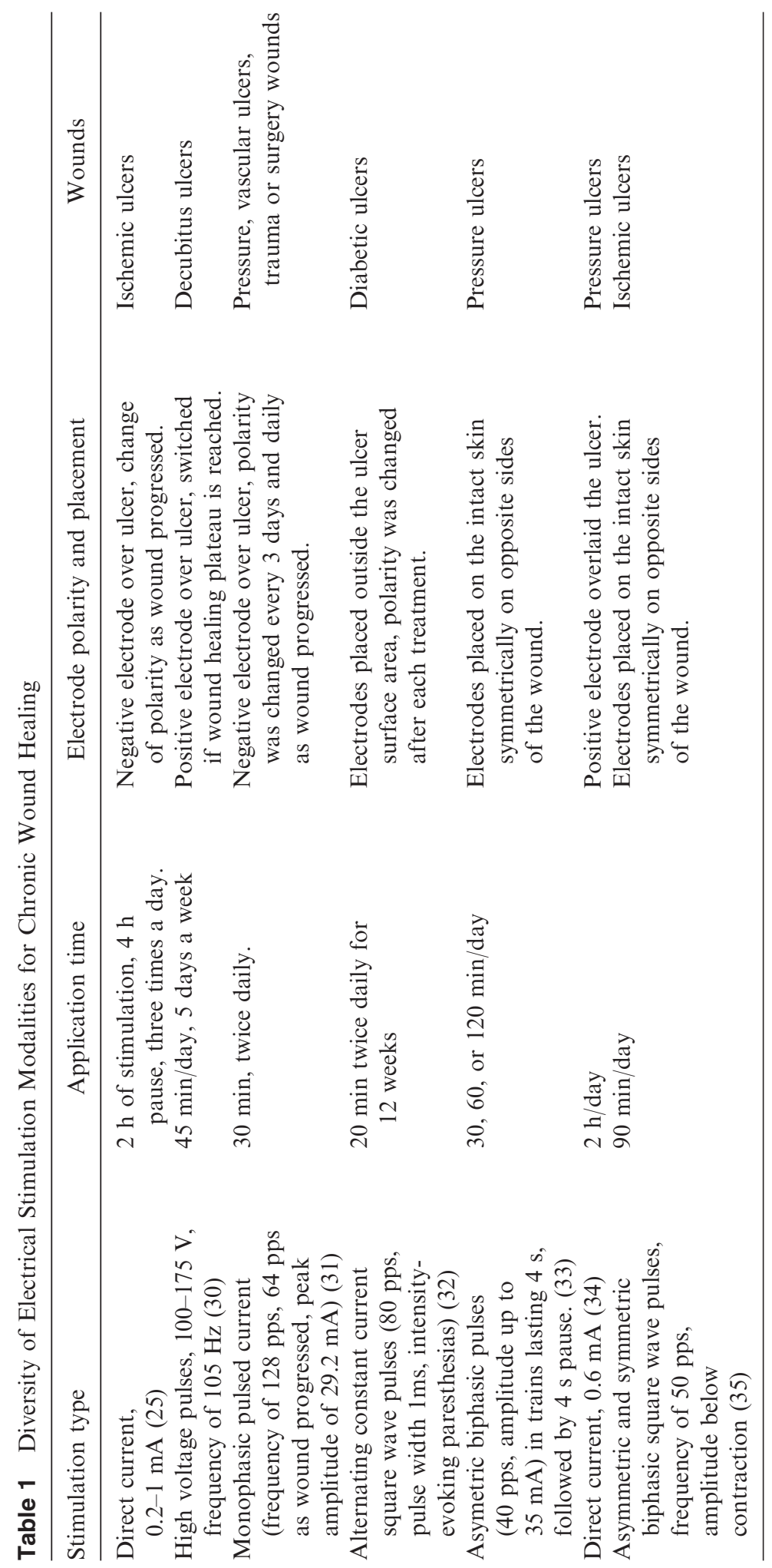


electrical properties are most effective and which wounds response to this best. In following subsections electrical waveforms used in electrical wound healing are divided into subsections: direct current, low-frequency pulsed currents, monophasic high-voltage pulses, and pulsed electromagnetic fields.

\section{A. Direct Current Electrical Stimulation}

In normal, uninjured human skin, a difference in ionic concentrations is actively maintained between the upper and lower epidermal layer, which can be measured as a difference of electrical potentials, ranging between 10 and $60 \mathrm{mV}$ on different locations on the body surface. The positive terminal of this so-called epidermal battery is located on the inside surface of the living layer of the epidermis (36). After wounding, when the skin layers are interrupted, the epidermal battery at the wound site is short-circuited, producing a conducting pathway, which allows ionic current to flow through the subepidermal region out of the wound and return to the battery by flowing through the region between the dermis and the living layer. The injury current (in $\mu \mathrm{A}$ range) can only flow, as along as wound surface is moist. The active role of endogenous electrical phenomena in wound healing is indirectly confirmed by the fact that the healing of wounds, the surface of which is kept moist, is more successful than in wounds that are left to dry out. Modeling of wound edge has shown relatively steep lateral voltage gradient across the edge, which means that the cells on the wound edge are situated in an electric field (34). Electric fields on order 100-200 mV/ $\mathrm{mm}$ have been measured lateral to wounds in mammalian epidermis.

Endogenous wound-induced electric fields present in the cornea plays role in the healing process by helping guide the cellular movements that close wounds. It has been shown that externally applied electrical fields of such "physiological" intensities can affect orientation, migration, and proliferation of cells (37), which are of key importance for healing, such as fibroblasts and keratinocytes (38-41). Several studies have confirmed that externally induced electrical fields with endogenous electrical conditions, positive electrode on the wound surface, and negative on the healthy skin around the wound, accelerate wound healing. Electrical currents were in range from $0.2 \mathrm{~mA}$ to $1 \mathrm{~mA}$. The application of negative electrode on wound surface was reported to have antimicrobial effect $(25,42)$ and was stipulated to be useful in initial stage of treatment.

\section{B. Low Frequency Pulsed Electric Currents}

Low-frequency pulsed electric current applications are quite popular in physical medicine. They are most commonly used for functional electrical stimulation to provoke involuntary muscle contraction for strengthening muscles atrophied by disuse and for eliciting functional movements in patients with motor disfunction $(43,44)$. Such electric current pulses are also known as tetanizing currents. Low-frequency pulsed electric currents were not applied only locally to the wound but also to areas quite distant to the wound. The two major distant locations were the spinal cord and acupuncture points (21). When low-frequency pulsed electric currents are applied locally both electrodes are placed on the healthy skin surrounding the wound. The amplitude of pulses is set to value just below visible tetanic contraction of surrounding muscles. This treatment modality is noninvasive and simple to use. The formation of chronic wounds is principally caused by an insufficient supply of oxygen and nutrients to the tissues due to poor blood flow. Daily use of low-frequency pulsed electric current stimulation was found to significantly increase partial oxygen tension $\left(\mathrm{pO}_{2}\right)$ around the chronic wound while no significant changes of $\mathrm{pO}_{2}$ were found when direct 
current electrical stimulation was used (45). Increase of $\mathrm{pO}_{2}$ during low-frequency pulsed electric current stimulation in patients with ischemic ulcers was caused by beneficial effects on the microcirculation. It is assumed that hypoxia or the release of metabolites during electrical stimulation due to insufficient blood flow and lack of oxygen represents stimuli for capillary growth.

\section{Monophasic High-Voltage Pulses}

Muscle is contracted at application of low pulse amplitudes and longer durations as well as at large amplitudes and shorter pulse durations. For the use of short high-voltage pulses no physiological explanation could be found. The positive electrode is placed over the wound and voltage set just below that capable of producing visible muscle tetanic contraction. The polarity of electrode over the wound was reversed if treatment reached the healing plateau. Reversing electrode polarity was successful when wounds were infected. Negative electrode placed on the wound has disinfection effect. It is reported that high-voltage stimulation improves blood flow and therefore facilitate wound healing $(30,46)$. It is hypothesized that high-voltage pulses stimulation restores sympathetic tone and vascular resistance below the level of the spinal cord lesion, thereby increasing the perfusion pressure gradient in the capillary beds. As such, high-voltage pulses stimulation could be used for preventing pressure ulcers (47).

\section{Pulsed Electromagnetic Fields}

Since experimental and clinical data suggested that exogenous electromagnetic fields (EMF) at low levels can have a profound effect on a large variety of biological systems, this led to the use of EMF signals in the treatment of large variety of diseases. It is successfully used clinically in all areas of bone fracture management $(48,49)$. The effect of noninvasive EMF on soft tissues is less well defined and it remains unclear though in vivo animal experiments, in vitro cell research and selected clinical studies confirmed accelerated wound healing. Markov and Pilla (27) in their detailed review of EMF stimulation of soft tissues discuss mechanisms of EMF treatment of nonhealing wounds.

\section{WOUND-HEALING QUANTIFICATION METHODS}

Despite the fact that different research groups have demonstrated that electrical stimulation can accelerate wound healing, it is still not widely used. Universal efficiency of electrical stimulation, diversity of small studies, unsuitable wound healing quantification methods, and not well established mechanisms that can explain how electrical stimulation affect the behavior of living cells and tissues render optimization of electrical stimulation difficult. Due to different quantification methods used, it is impossible to make a quantitative analysis of the comparative advantages and disadvantages of different treatment modalities. In order to enable quantification and comparison of treatment efficacy, uniform measure of wound healing needs to be generally accepted, which ideally would fulfill the following criteria: simple calculation, suitable for statistical handling, transparency-evident physiological meaning, employability for different wound types, sizes, shapes, and healing and/or nonhealing courses. Quantitative measurement of wound healing should enable service providers to assess, improve, and individualize the treatment given to each wound patient. In order to correctly quantify wound healing wound has to be periodically assessed and wound healing process dynamics has to be considered. 


\section{A. Wound Status Assessment}

Wound assessments provide the foundation of the plan of care and are the only means of determining the effectiveness of the treatment. Regular reassessments are crucial in clinical trials and practice to provide the care provider an insight into the time course of the wound healing by comparing the series of wound data collected over time (50). Documented reassessments can be reanalyzed for treatment optimization. Chronic wound assessment requires quantification of multiple parameters of the wound and surrounding tissue. Lazarus et al. (51) proposed guidelines for wound assessment. They listed attributes that are clues to the cause, pathophysiology, and status of the wound. Clinical assessment should include wound history, anatomic location, stage, size, sinus tracts, undermining, tunneling, exudate or drainage, necrotic tissue, presence or absence of granulation tissue, and epithelization. Intact skin surrounding the wound should be assessed for redness, warmth, induration or hardness, swelling, and any obvious signs of clinical infection (52).

Assessment of wound status should begin with the extent of the wound. Because the extent of the wound changes with time, it requires periodic assessments. There are several techniques that may be employed to assess wound extent. To be clinically acceptable, the assessment of chronic wound healing has to be noninvasive, inexpensive, and practical enough to be regularly used by clinicians. In several past years number of studies to enhance tools for monitoring healing steeply increased. Studies were primarily focused on periodical noncontact wound status assessments and their documenting. Noncontact systems for wound status assessment base on wound size measurements, mostly on wound area. Systems are in various phases of testing on plaster molds and animal wound models:

Structured lighting pattern captured on a digital photograph of a wound can be used to calculate the area and volume of debrided wound $(53,54)$.

Computer assist planimetric methods using digitalized tracings of the wound (55).

Three-dimensional laser imaging system for wound area and volume assessment (56).

Digital imaging technique and planimetry for wound area assessment (57).

Laser scanner for wound topography measurement and calculation of wound volume and area assessment (58).

The literature reveals that assessment of wound area, wound perimeter, or mutually perpendicular diameters (largest diameter of the wound and diameter taken at right angle to the largest one) are most frequently used. Classical wound volume and depth assessment techniques are invasive because we have to insert our measuring device or material (dental moulds) into the wound (59). Besides the disturbance of the wound, the volume or depth can be underestimated because of invisible edge at the bottom of the wound and degenerative tissue, which fills up the wound. Invasive measurement methods could interfere with healing; therefore, they are generally avoided. Noncontact methods require expensive equipment such as stereoscopy, MRI (60) or above described novel techniques and are rarely used. Since wounds are often irregular in shape and heal asymmetrically, different estimates of wound area are used. Acetate tracings can provide the most accurate description of wound area and perimeter but require manual or computer planimetry. Automatic wound contour detection methods from digitized images of wounds could in future simplify wound area assessment (61). Estimates of wound area can be calculated from the product of two mutually perpendicular perimeters or by calculation of the area of a circle or ellipse from measured diameters. Surface area can also be estimated by simply comparing ulcers to predrawn circles or ellipses of known area. Results of studies suggest that simple and cost- 
effective wound area measurement techniques such as ellipse estimate may confidently be used to monitor healing in clinical settings (62-64).

Another wound status assessment possibility is scaling systems where scaling of wound status is determined by one or several indicators of wound healing such as wound extent, necrosis, surrounding skin color, peripheral tissue edema and induration, granulation tissue, epithelialization, infection, drainage, eschar and exudates. Shea, in 1975 was one of the first to propose a standard wound classification system (65). It was based mainly on wound depth and did not focus on presence or absence of infection. Additionally, the system did not mention ischemia as a co-morbid factor. Because of these limitations, several classification systems have been proposed since then (66). There are three widely accepted criteria used to classify the stages of ulcers. The most widely used pressure ulcer scaling system is the four stage system developed by the National Pressure Ulcer Advisory Panel (NPUAP), Merck Manual for decubitus ulcers, and Wagner's Classification system for foot ulcers. NPUAP warns that staging should not be used to determine progress toward the wound healing, because stage IV pressure sore is always a stage IV ulcer no matter how it is healing. For this reason several classification systems have been proposed, that are responsive to changes during wound healing. These systems are still in various stages of testing, but two of the most promising appear to be the seven point categorical Sessing scale (67) and Pressure Ulcer Scale for Healing (PUSH) (68). Recently PUSH system was demonstrated on clinical data to be valid and sensitive measure of pressure ulcer healing (69) with the components of length times width, exudates amount, and tissue type, though further testing is needed to confirm these findings. Scaling systems are widely used as a wound assessment alternative, as they are practical for daily monitoring. However it is still not clear if it is appropriate to use them for the follow-up of changes in wound healing. The small number of stages makes them easy to use but at the same time makes them not sensitive enough for wound healing progress description (70). Based on above considerations wound extent should be evaluated for monitoring wound status when progress toward the wound healing has to be determined.

\section{B. Wound-Healing Process Dynamics}

If assessment of wound extent is a quantitative value (a scalar) and is periodically assessed, linear or nonlinear regression can reveal wound healing dynamics over time. The majority of researchers use measures of wound extent that incorporate only wound area, while wound volume, depth, and perimeter are rarely used. Gilman (71) defined a measure of wound extent that incorporated wound area and perimeter and was termed the advance of the wound margin toward the wound center. Wound extent is mostly defined either as absolute or normalized wound area. Normalized wound area is calculated as wound area divided by the initial wound area and multiplied by 100. Dynamics of the healing processes followed either by measuring absolute or normalized wound area over time are the same.

Researchers generally use either linear or exponential models to present time course of wound-healing process. Both models are distinguished for small number of parameters, however, neither of models has an adequate physiological basis. Most prominent disadvantage of the linear model is that it sets no limit to wound area. Recent research revealed that time course of wound area had in $51 \%$ of wounds included in the study decay exponential shape after initial delay longer than 3.5 days. In $40 \%$ of wounds the delay was more than 7 days and in $26 \%$ of wounds the delay was more then 14 days. Exponential model correctly described only $49 \%$ of time courses of wound area during healing. To consider the observed initial delay of healing a delayed exponential model was proposed for the most general mathematical model of chronic wound-healing dynamics (72). An example 
of such wound-healing dynamics is presented in Fig. 1 and a mathematical description of the delayed exponential model is given by Eq. (1),

$$
\hat{S}(t)= \begin{cases}S_{D E X} & 0 \leq t<T_{D E X} \\ S_{D E X} e^{-\theta_{D E X}\left(t-T_{D E X}\right)} & t \geq T_{D E X}\end{cases}
$$

where $\hat{S}(t)$ is the estimated wound area in percent of initial wound area and three parameters $S_{D E X}, \theta_{D E X}$ and $T_{D E X}$ describe the wound healing dynamics. Parameter $S_{D E X}(\%)$ estimates initial wound area, and parameter $\theta_{D E X}\left(\right.$ day $\left.^{-1}\right)$ defines the time constant of exponent function and time delay of the healing process is defined by parameter $T_{D E X}$ (day). It has been demonstrated that this model has good predictive capability and in this capacity can be used to predict time needed to complete wound closure after at least 4 weeks of consecutive weekly measurements of wound area. Such model may be very useful in clinical trials, where not all wounds included in the study close within the designated study period.

Incorporating wound shape information (through wound perimeter) in wound extent measure did not improve wound healing dynamics description (73). Since it is easier to measure wound area than wound area and perimeter, estimation of wound healing dynamics from regular wound area measurements is preferred.

\section{Wound-Healing Rate Definition}

In spite of the evident need for uniform measure of wound healing rate, several measures have been employed in literature to date. The first group of wound healing rate measures base either on wound size assessments the beginning of the observation period and at its end or on periodical wound size assessments in the observation period. Wound healing rate was estimated as percentage reduction of wound area in 4 weeks (74), as percentage reduction of wound area in 12 weeks (32), as percentage reduction of wound area per day in observation

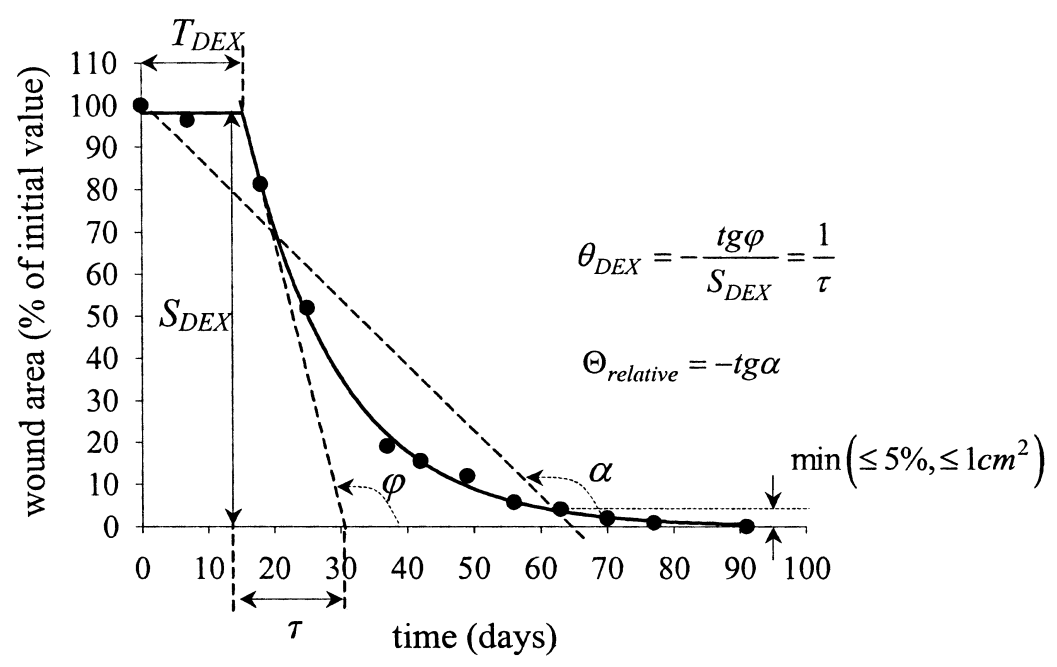

Figure 1 Example of following wound area and application of the delayed exponential model to normalized data. $\mathrm{S}_{\mathrm{DEX}}, \theta_{\mathrm{DEX}}, \mathrm{T}_{\mathrm{DEX}}$ are calculated parameters of the delayed exponential model, and $\Theta_{\text {relative }}$ is a measure of wound healing rate. (-) Fitted delayed exponential model; (•) normalized wound area (from Ref. 73). 
period (75), as average percentage reduction of wound area per week in 4 weeks (31), as average of the sequentially computed weekly healing rates (normalized difference between two sequential measurements) in percent of initial wound area per week (35), as time needed to complete wound closure (76), as average linear healing of the wound edge toward the center after 2 weeks in distance per day (77), and as time constant of exponential function fit to weekly wound area measurements (33). The second group of wound healing rate measures base on changes in scorings, assessed using classification systems, over time. Wound-healing rate was defined as average change in Sessing scale between two consecutive scorings assessed twice per week (67), and as linear regression to PUSH values in the initial, second, fourth, sixth, and eighth week of the observation period $(68,78)$. The majority of authors have used a measure of the wound-healing rate that assumes linear wound extent variation over time. This assumption is misleading since dynamics is nonlinear regardless of how the wound extent is measured.

The goal of wound care is complete wound closure. Therefore, wound healing rate should describe time needed to wound closure and it should be irrespective of wound aetiology, location, and treatment. Wound-healing rate expressed as absolute area healed per day tends to exaggerate the healing rates of larger wounds and healing rate expressed and as percentage of initial area healed per day tends to exaggerate the healing rates of smaller wounds. Wound-healing rate should not be affected by wound size, when wounds of differing sizes are compared. Only wound-healing rate expressed as the advance of the wound margin toward the wound center per day is not influenced by initial wound size (73). The wound-healing rate defined as the advance of wound margin towards the wound center is defined as

$$
\Theta=2 \frac{S_{0}}{p_{0}} \frac{1}{T} \mathrm{~mm} / \mathrm{day}
$$

where $S_{0}$ is the initial wound area, $p_{0}$ is the initial perimeter, and $T$ is the time to complete wound closure. Positive value of wound-healing rate indicates healing wounds and negative value of the wound-healing rate is the estimate of wound growth velocity towards its double initial area. For the wound healing rate $\Theta$ to be appropriately calculated, we have to follow the wound-healing process till the complete wound closure. Because clinical trials are financially and time limited, the time to complete wound closure has to be predicted from collected wound extent measurements in observation period, which may be much shorter than time to the complete wound closure. However, prediction of the time to complete wound closure could help clinicians to early detect not efficiently treated wounds. To predict time to complete wound closure wound extent has to be periodically measured and a known model fitted to the collected data. From calculated values of model parameters, the time to complete wound closure can be calculated. Since exponential function reaches its asymptote at infinite time, wound can be defined to be closed when the mathematically predicted wound area is smaller than $5 \%$ of initial value and at the same time smaller than $100 \mathrm{~mm}^{2}$.

\section{CLINICAL STUDIES}

During more than a decade lasting clinical use of electrical stimulation, data concerning patients, wounds, and their treatment were assessed and documented. The Ethical Committee of the Republic of Slovenia approved the study. The patients were examined by physician for an initial assessment of their wound status and relevant factors. The experimental procedure was explained to them and all patients agreed to participate in the 
study by signing an informed consent form. Together, 266 patients with 390 wounds were recorded in our computer database up to date. Unfortunately, many patient and wound data are missing, and not all wounds were followed regularly or until the complete wound closure, which is relatively common problem in clinical trials. Wound case inclusion criteria (initial wound area larger than $1 \mathrm{~cm}^{2}$ and at least four weeks of wound healing process follow-up) were fulfilled in 300 wound cases (214 patients). Our study enrolled wounds of various aetiologies (e.g., vascular ulcerations, amputation wounds, pressure ulcers, neuropathic ulcerations), locations, and different treatments in patients with different primary diagnoses (e.g., spinal cord injury, diabetes mellitus, sclerosis multiplex, vascular diseases).

All patients received conservative treatment of their chronic wounds. The conservative treatment included initial selective debridement, the application of a new standard dressing to the chronic wound two or more times per day, as needed, and broad-spectrum antibiotics in cases of infection, which were rather rare. Fifty-four $(18.0 \%)$ wounds received only conservative treatment. In addition to the conservative treatment, $23(7.7 \%)$ wounds received sham treatment, where electrodes were applied to the intact skin on both sides of the wound for two hours daily and connected to stimulators, in which, however, the power source was disconnected and they delivered no current. Two different modes of electrical stimulation were used: direct and biphasic current. Forty-two $(14.0 \%)$ wounds were stimulated with direct current of $0.6 \mathrm{~mA}$ for $0.5 \mathrm{~h}, 1 \mathrm{~h}$, or $2 \mathrm{~h}$ daily. Positive stimulation electrode overlaid the wound, surface and negative electrode was placed on the intact skin around the wound, or both electrodes were placed on the healthy skin at the wound edge across the wound, one of them being positive and the other negative. We have pooled different electrode placements in direct current stimulation group in spite of the difference in effectiveness of direct current stimulation (34). We did this for two reasons: in literature both electrode placements were shown to accelerate chronic wound healing; and in this way we kept otherwise small direct current stimulation group of wounds at the size that allowed us statistical analysis. One hundred eighty-one $(60.3 \%)$ wounds were stimulated with biphasic, charge-balanced current pulses (79) for $0.5 \mathrm{~h}, 1 \mathrm{~h}$, or $2 \mathrm{~h}$ daily with electrodes placed on both sides of the wound. The pulse duration was $0.25 \mathrm{~ms}$ and at a repetition rate of $40 \mathrm{~Hz}$. The 4-s stimulation trains were rhythmically alternated with pauses of the same duration. The pulsed currents produce tetanic contraction of the stimulated tissue, which is kept at a minimum level (adjusted by the stimulation amplitude, usually at 15 to $25 \mathrm{~mA}$ ) to prevent mechanical damage of the newly formed tissue (Fig. 2).

The currents were applied across the wound by a pair of self-adhesive skin electrodes (Encore TM Plus, Axelgaard Manufacturing Co. Ltd.) attached to the healthy skin at the edge of the wound. In direct stimulation group, where positive stimulation electrode overlaid the wound surface, the wound surface was covered with sterile gauze, soaked in physiological solution, on top of which a conducting rubber electrode was applied. This assured uniform current distribution throughout the entire wound area. Four self-adhesive electrodes were attached to the intact skin around the wound, representing the ring-shaped negative electrode. At the beginning of our study in 1989, wounds were randomly assigned into four treatment groups: conservative treatment, sham treatment, biphasic current stimulation, and direct current stimulation. Since Jerčinović et al. (33) showed that stimulated wounds were healing significantly faster than conservatively or sham treated wounds, it was not ethical to keep including patients in those groups. After Karba et al. (34) reported that electrical stimulation with direct current is effective only if positive electrode is placed on the wound surface, which is an invasive method, only stimulation with biphasic current pulses was used. Therefore, the group of patients stimulated with biphasic current pulses is larger than other groups of patients. 


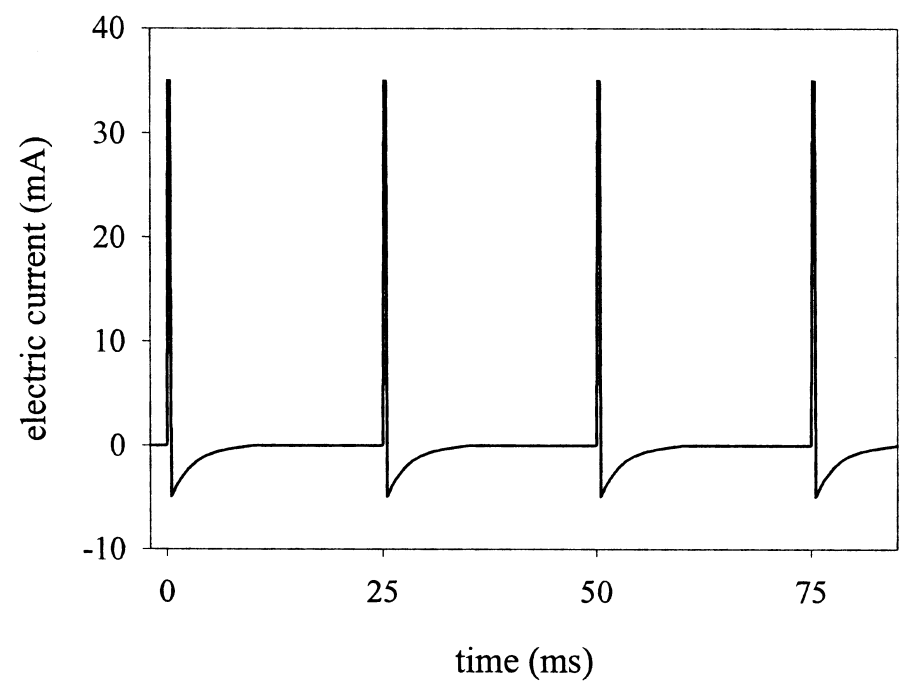

Figure 2 Electrical properties of biphasic electrical current stimulation.

For the evaluation of the efficacy of particular treatment modality or for the evaluation of the influence of wound and patient attributes on wound healing, wound area was periodically followed. Wound shape was approximated with an ellipse and thus it was enough to periodically follow mutually perpendicular diameters of the wound. From wound diameters, the wound area, perimeter, and width-to-length ratio were calculated. As alternative measure of wound extent we used the four-stage Shea grading system (65). Wound depth and grade were collected only at the beginning of treatment. Wounds were treated daily till complete wound closure. If wound did not completely heal within the observation period, the patient continued his treatment at home, but follow-ups were discontinued because the reliability of the home treatment was questionable. Among 300 wound cases, in 174 cases wounds were followed untill complete wound closure, while in 126 cases time to complete wound closure was estimated $(72,73)$. No significant difference between actual time to complete wound closure and estimated one from wound extent measurements in observation period longer than 4 weeks was observed. Because time to complete wound closure was found dependent on initial wound extent, a measure of the wound healing rate an average advance of the wound margin towards the wound centre was used. In Table 2 wound, patient, and treatment data collected in our computer database are listed. These data were selected to be attributes of chronic wound description. All listed attributes except wound extent were collected at the beginning of wound treatment. In addition, wound extent was followed weekly during the observation period or until the complete wound closure.

Plotting percentage of healed wounds against the time elapsed from the beginning of the treatment (Fig. 3) revealed differences between the four treatment groups. Electrically stimulated wounds healed at higher rate and extent than other wounds. Over $90 \%$ of electrically stimulated wounds healed within 60 weeks, while only $70 \%$ of sham treated wounds and $72 \%$ of conservative treated wounds healed within the same period. The wound-healing rate revealed significant differences between four treatment groups. Results of Kolmogorov-Smirnov Two Sample nonparametric test comparing treatment modalities ( $p$ values) revealed that wounds treated with biphasic current stimulation healed signifi- 
Table 2 Wound, Patient, and Treatment Data Categories Collected in a Database During More Than a Decade of Using Electrical Stimulation at the Institute of the Republic of Slovenia for Rehabilitation
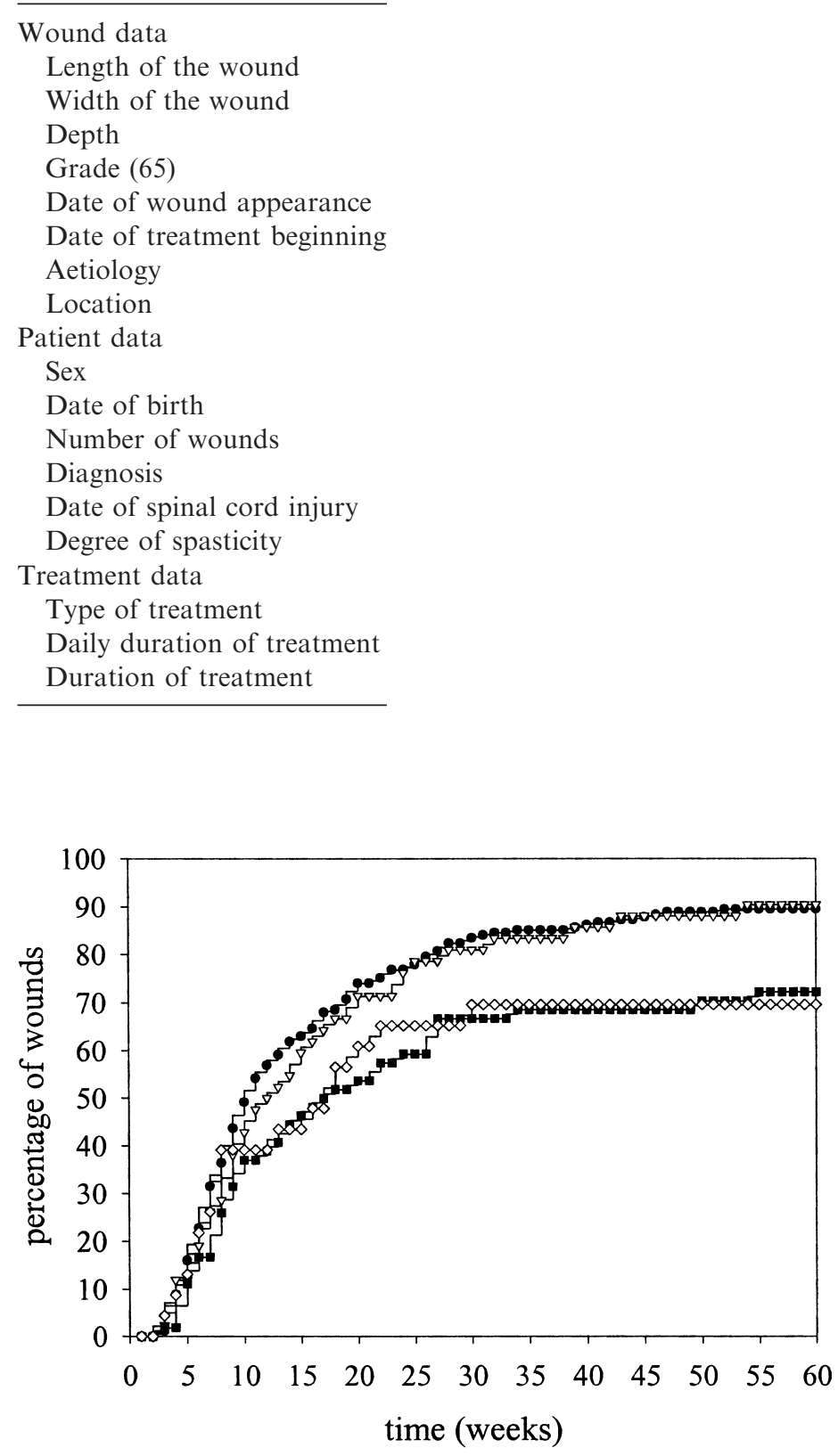

Figure 3 Percentage of healed wounds against time elapsed from beginning treatment for four treatment modalities: $(--)$ biphasic current stimulation; $(-\nabla-)$ direct current stimulation; $(-\boldsymbol{-}-)$ conservative treatment; $(-\downarrow)$ ) sham treatment (from Ref. 83). 
cantly faster than conservative or sham treated wounds. No significant difference was found in healing rates between wounds treated with direct current and wounds treated with biphasic current pulses. Difference in healing rates between direct current and conservative or sham treatment was considerable, in favor of direct current, although it was not significant. Conservative or sham treated wounds healed at the same rate.

\section{A. Prognostic Factors in the Prediction of Wound Healing}

However, dynamics of the wound healing process does not depend only on the type of the treatment, but depends also on wound and patient attributes. The aims of our study were to determine the effects of wound, patient, and treatment attributes on wound-healing process and to propose a system for prediction of the wound healing rate. Only a limited number of groups have investigated wound and patient attributes which affect chronic wound healing (74-76,80,81) and none of them incorporates electrical stimulation as the chronic wound treatment modality. The quantity of available data from our clinical study of electrical wound healing permitted us to employ statistical tools and artificial intelligence methods for analysis of the healing process itself, as well as of the effects of different therapeutic modalities. In the first step of our analysis we determined which wound and patient attributes play a predominant role in the wound-healing process. Then we discussed the possibility to predict wound-healing rate at the beginning of treatment based on initial wound, patient and treatment attributes. Finally we discussed the possibility to enhance the wound healing rate prediction accuracy by predicting it after a few weeks of wound healing follow-up.

\section{Wound-Healing Rate Prediction from the Model Wound-Healing Dynamics}

We determined that the wound area variation over time has a delayed exponential behavior. Delayed exponential equation is thus the structure of mathematical model of the wound healing process and by fitting this model to a particular chronic wound case; parameters of the model are calculated. At least four measurements of wound area (performed in at least three weeks) are needed before parameters of mathematical model can be estimated. From parameters of mathematical model the time to complete wound closure was estimated.

According to Eq. (2) the estimated wound healing rate was calculated. We found that the estimated wound healing rate after at least 4 weeks of wound follow-up did not differ significantly from the actual one $(p \geq 0.2)$. However, if a wound extent was followed less than 4 weeks the difference was found to be significant. In clinical trials 4 weeks is a short period, but in clinical practice a shorter time for treatment outcome prediction may be required.

\section{Statistical Analysis}

Distribution of the wound healing rate was not normal; non-parametric statistical analysis was therefore employed. To determine differences in distribution of quantitative attributes in groups formed by qualitative attributes we used the Kruskal-Wallis one-way analysis of variance. To test relationship of qualitative attributes, we used a chi-square test. To determine if two quantitative attributes are correlated, we used the Spearman correlation test $(r s=$ Spearman correlation coefficient, $p=$ probability of being wrong in concluding that there is a true association between the variables, and $n=$ number of cases).

Statistical analysis revealed that the time to complete wound closure is correlated to wound extent attributes, area $(r s=0.428, p<0.001)$, and grade $(r s=0.388, p<0.001)$. The wound-healing rate is not correlated to initial area, perimeter, or width to length ratio but is moderately correlated to wound grade $(r s=-0.237, p<0.001, n=281)$. Wounds of 
higher grade were healing slower. Wound grade also tends to increase with increasing initial wound area $(r s=0.292, p<0.001, n=281)$.

Time elapsed from wound appearance to the beginning of treatment was modestly correlated to wound grade $(r s=0.181, p=0.005, n=243)$, which can indicate that wounds should be treated as soon as they appear. Therefore it was also expected that the wound not appropriately treated for a long period would heal slowly (negative correlation coefficient when comparing AppearStart with the wound healing rate) $(r s=-0.215, p<0.001$, $n=243)$. A small initial wound area $(r s=-0.261, p<0.001, n=178)$ of wounds that appeared a long time after spinal cord injury (injuryappear), is probably a result of better patients self care.

Wounds on trochanter healed significantly slower $(p<0.030)$ than wounds on other locations. Locations did not differ with respect to grade $(p=0.236)$ but they differed with respect to area $(p<0.001)$, revealing significantly greater wounds on locations trochanter and sacrum than on gluteus or other locations. Wounds on trochanter, gluteus and sacrum were all pressure ulcers. Patients with wounds on sacrum or trochanter were significantly younger $(p<0.010)$ than patients with wounds on other locations.

Wounds of geriatric (healing rate $=0.271 \mathrm{~mm} /$ day) and traumatic patients were healing significantly faster $(p=0.005)$ than wounds of patients with other diagnosis: spinal cord injury $(0.173 \mathrm{~mm} /$ day $)$, vascular insufficiency $(0.171 \mathrm{~mm} /$ day $)$, diabetes mellitus $(0.102 \mathrm{~mm} /$ day) and multiple sclerosis $(0.138 \mathrm{~mm} /$ day $)$. We found diagnosis strongly related to wound aetiology $(p<0.001)$.

Electrically stimulated wounds healed at higher rate and extent than other wounds. Over $90 \%$ of electrically stimulated wounds healed within 60 weeks, while only $70 \%$ of sham treated wounds and $72 \%$ of conservative treated wounds healed within the same period. It was found that wounds treated with biphasic current stimulation healed significantly faster than conservative $(p=0.031)$ or sham $(p=0.008)$ treated wounds. No significant difference $(p=0.365)$ was found in healing rates between wounds treated with direct current and wounds treated with biphasic current pulses. Difference in healing rates between direct current and conservative $(p=0.085)$ or sham treatment $(p=0.056)$ was considerable, in favor of direct current, although it was not significant. Conservative or sham treated wounds healed at the same rate $(p=0.607)$.

Wounds stimulated by biphasic current for $2 \mathrm{~h}$ daily healed at the same healing rate as those stimulated for $0.5 \mathrm{~h}$ daily, while wounds stimulated for $1 \mathrm{~h}$ daily healed significantly ( $p=0.017$ ) faster than wounds stimulated for $2 \mathrm{~h}$ or $0.5 \mathrm{~h}$ daily. A lack of wound cases stimulated for $1 \mathrm{~h}$ daily $(n=13)$ renders this result statistically unreliable. Further study should be performed to optimise daily duration of electrical stimulation.

\section{Machine Learning Approach to Wound-Healing Rate Prediction}

From results of statistical analysis reported above, it is obvious that the wound healing rate is directly dependent on wound treatment and wound grade, while interactions of other wound and patient attributes on the wound healing rate are not easy to determine. Prognostic factors of wound healing are rarely analyzed in the literature and our study was first attempt to incorporate electrical stimulation as the chronic wound treatment modality. We employed tree learning algorithms to build regression and classification trees to predict the wound healing rate based on initial wound, patient and treatment data. We tested several algorithms for attribute selection among which RReliefF (82) for regression tree generation was found to be the most effective (83). For models in leaves of the tree, the most appropriate were linear equations. A stopping rule of minimal five wound cases in a leaf was used. Since the sample size $(n=300)$ was moderate, the 10 -fold cross-validation was 
used as the error estimation method. The accuracy of regression trees was measured as relative squared error (relative error) (84), which is always nonnegative and usually less than 1 . Trees with relative error close to 0 produce good prediction of the wound healing rate, and trees with the relative error around 1 or even greater than 1 produce poor prediction.

Attributes partitioning powers calculated using machine learning algorithm RReliefF revealed that initial wound area, followed by patient's age and time from wound appearance to treatment beginning are the most prognostic attributes, followed by wound shape (widthto-length ratio), location of wound, and type of treatment. Generated regression trees with linear equations in leaves for the wound healing rate prediction at the beginning of treatment had relative squared error greater than one, which means that resulting regression trees are not usable. Adding the model estimate of the wound-healing rate in a set of variables used for regression tree generation reduced relative error of generated regression tree. Woundhealing rate estimated only after 1 week of wound area follow-up reduced the relative error of generated regression tree to 0.64 , similarly after 2 weeks to 0.35 , after 3 weeks to 0.18 (Fig. 4) and after 4 weeks of follow-up to 0.09. Afterwards relative error was slowly decreasing to 0.06 in 6 weeks of follow-up. After 5 weeks, the wound-healing rate predicted by regression tree was equal to the healing rate estimated by the delayed exponential model. The predicted wound-healing rate in shorter period in addition depends on wound, patient, and treatment attributes. Rough estimation of wound healing rate can be determined only after 2 weeks of wound healing follow-up. Type of treatment is indirectly included in regression trees as daily duration of treatment, which was zero in case of conservative or sham treated wounds. Important prognostic attributes are wound area, grade, shape (width to length ratio), patients age, elapsed time from spinal cord injury to wound appearance, and elapsed time from wound appearance to the beginning of treatment.

Considering also prognostic factors: deep vein involvement, ankle/brachial pressure index, liposclerosis, edema, exudates and granulation, which are reported in the literature $(74,80)$ as prognostic factors, our prediction might be even more accurate. Regression trees in combination with prediction capability of delayed exponential model of wound healing dynamics are basis for the prognostic system for prediction of chronic wound healing rate.

\section{B. Quantitative and Qualitative Changes in the Tissue After Electrical Wound Healing}

The proof weather a method of treating wounds is successful is a matter of histological analyses of the affected soft tissue before and after treatment $(85,86)$. Reports of histological

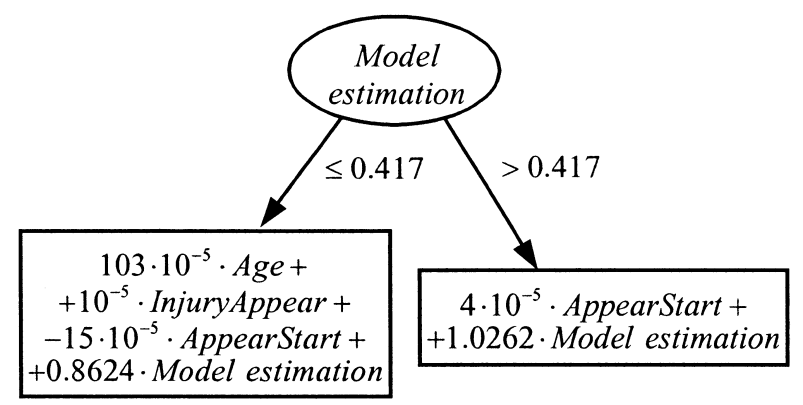

Figure 4 Regression tree with linear equations in leaves for prediction of wound healing rate after 3 weeks of treatment (from Ref. 83). 
analyses of electrical wound healing are rare especially in clinical trials. At the Institute of Rehabilitation a histological study of electrical wound healing was done recently. The study enrolled 50 patients with spinal cord injury, suffering from decubital ulcers of III degree according to Shae scale (65) in the sacral area. A half of wounds were treated according to described biphasic electrical stimulation treatment and another half received only conservative treatment. In five patients from each group a qualitative and a quantitative histological analyses of the tissue samples (about $4 \mathrm{~mm}^{3}$ ) taken from the wound, on the line between the wound edge and freshly formed scar, were performed before the beginning of treatment and after around 2 months, when the formed scar formed during the electrical stimulation was of considerable size.

Wound healing was followed as described in above clinical study section. Significantly faster healing of wounds in electrically stimulated group was observed. The histological preparations were analyzed by a quantitative stereological method. Content of surface collagen in the preparations stained according to Masson and the surface density of blood vessels was determined in the immunohistochemically stained preparations. The surface percentage of collagen was determined by using test system M-42 and the number of blood vessels per surface unit by a semiautomatic IBAS 1000 image processing and analysis system. Wounds treated by electrical stimulation had lower inflammatory response, higher collagen density as well as more intense process of angiogenesis. In electrically healed group collagen density increased in average $23 \%$, while in the control group decreased by $2 \%$ of the initial surface in two months time period. The area density of blood vessels was higher in electrically stimulated wounds, and in poststimulation period the blood vessels were found to be reaching essentially higher towards the wound surface than in the nonstimulated wounds, in fact, almost as far as the crust.

In stimulated wounds endothelial cells were flat, the blood vessels lumina broad with erythrocytes clearly visible within them. In control group endothelial cells were thickened, cubically shaped, with round nuclei, and no erythrocytes were visible within blood vessels lumina. Also previous in vitro studies $(37,87)$ reported flatter endothelial cells exposed to electromagnetic or electric field, which are of cubic shape when not exposed to the field.

The study showed that the intercellular substance is dominated by fibrin whereas more collagen was found in the sample preparations of electrically healed wounds. The conclusion is that electrical stimulation may exert the release of mediators responsible for the increase in collagen synthesis in fibroblasts or the shrinking of myofibroblasts. Furthermore the study showed that electrical healing has a favorable effect on blood circulation in the wound, improves blood circulation in the tissue surrounding the wound and improves the quality of posttreatment scar.

\section{CONCLUSIONS}

One of the largest clinical studies of electrical wound healing and its outcomes are presented. Electrically stimulated wounds healed faster and at greater percentage than conservative or sham treated wounds. We noticed slightly slower healing of wounds treated with direct current than wounds treated with biphasic current, but both treatment modalities accelerate wound healing of chronic wounds. Histological analysis confirmed positive effects of biphasic current electrical stimulation, such as improved blood circulation in the wound and surrounding tissue, as well as improved posttreatment scar.

Electrical treatment regime should in future be optimized regarding electrical parameters used and daily duration of treatment. However it should also be determined which treatment regimes apply best for different wound aetiologies. Unified wound-healing 
quantification and documentation will enable researchers to optimize electrical wound healing and promote it in clinical practice.

It was demonstrated that wound healing process can be weekly followed by simple wound area and perimeter measurements. In future non contact measuring devices will simplify wound extent measurement and documentation. For accurate wound-healing rate estimation, wounds should be followed at least 4 weeks, while using generated regression trees follow-up can be reduced to 3 weeks for wound healing estimation with relative error 0.18 . Therefore, the wound-healing rate or the time to complete wound closure can be estimated after 3 weeks of treatment, which can help to formulate appropriate management decisions, reduce the cost, and orient resources to those individuals with poor prognosis.

\section{ACKNOWLEDGEMENTS}

The authors are in depth to Prof. Damijan Miklavčič for his valuable help during the chapter preparation. This work was supported by Slovenian Ministry for Education, Science and Sport.

\section{REFERENCES}

1. Waldorf H, Fewkes J. Wound healing. Adv Dermatol 1995; 10:77-97.

2. Dagher JF. Cutaneous Wounds. Mount Kisco, NY: Futura Publishing Company, 1985:99-220.

3. Sanders SL. Pressure ulcers, Part 1: Prevention strategies. J Am Acad Nurse Pract 1992; 4(2):6370 .

4. Whiteneck GG, Charlifue SW, Frankel HL, Fraser MH, Gardner BP, Gerhart KA. Mortality, morbidity, and psychosocial outcomes of persons spinal cord injured more than 20 years ago. Paraplegia 1992; 30(9):617-630.

5. Richardson RR, Meyer PR. Prevalence and incidence of pressure sores in acute spinal-cord injuries. Paraplegia 1981; 19(4):235-247.

6. Mawson AR, Biundo JJ, Neville P, Linares HA, Winchester Y, Lopez A. Risk-factors for early occurring pressure ulcers following spinal-cord injury. Am J Phys Med Rehabil 1988; 67(3):123127.

7. Fuhrer MJ, Garber SL, Rintala DH, Clearman R, Hart KA. Pressure ulcers in communityresident persons with spinal-cord injury-prevalence and risk-factors. Arch Phys Med Rehabil 1993; 74(11):1172-1177.

8. Goodman CM, Cohen V, Armenta A, Thornby J, Netscher DT. Evaluation of results and treatment variables for pressure ulcers in veteran spinal cord-injured patients. Ann Plast Surg 1999; 42(6):665-672.

9. Kantor J, Margolis DJ. Treatment options for diabetic neuropathic foot ulcers: A costeffectiveness analysis. Dermatol Surg 2001; 27(4):347-351.

10. Braddock M, Campbell CJ, Zuder D. Current therapies for wound healing: electrical stimulation, biological therapeutics, and the potential for gene therapy. Int J Dermatol 1999; 38(11):808817.

11. Martin P, Hopkinson-Woolley J, McCluskey J. Growth factors and cutaneous wound repair. Prog Growth Factor Res 1992; 4(1):25-44.

12. Kunimoto BT. Growth factors in wound healing: the next great innovation? Ostomy Wound Manage 1999; 45(8):56-64.

13. Limova M. New therapeutic options for chronic wounds. Dermatol Clin 2002; 20(2):357- + .

14. Beele H. Artificial skin: Past, present and future. Int J Artif Organs 2002; 25(3):163-173.

15. Kannon GA, Garett AB. Moist wound healing with occlusive dressings. A clinical review. Dermal Surg 1995; 21:583-590.

16. Lucas C, Coenen CHM, de Haan RJ. The effect of low level laser therapy (LLLT) on stage III 
decubitus ulcers (pressure sores); a prospective randomised single blind, multicentre pilot study. Lasers in Medical Science 2000; 15(2):94-100.

17. Lagan KM, McKenna T, Witherow A, Johns J, McDonough SM, Baxter GD. Low-intensity laser therapy/combined phototherapy in the management of chronic venous ulceration: A placebo-controlled study. J Clin Laser Med Surg 2002; 20(3):109-116.

18. Brown M. Ultrasound for wound management. In: Gogia Prem P, ed. Clinical Wound Manage. Thorofare: SLACK, 1995:197-206.

19. Nessbaum EL, Biemann I, Mustard B. Comparison of ultrasound/ultraviolet-C and laser for treatment of pressure ulcers in patients with spinal cord injury. Phys Ther 1994; 74(9):812-825.

20. Kalani M, Jorneskog G, Naderi N, Lind F, Brismar K. Hyperbaric oxygen (HBO) therapy in treatment of diabetic foot ulcers - Long-term follow-up. J Diabetes Complications 2002; 16(2): $153-158$.

21. Vodovnik L, Karba R. Treatment of chronic wounds by means of electric and electromagnetic fields, Part 1 Literature review. Med Biol Eng Comput 1992; 30:257-266.

22. Markov MS. Electric-current and electromagnetic-field effects on soft-tissue-implications for wound-healing. Wounds-A Compendium of Clinical Research and Practice 1995; 7(3):94-110.

23. Markov MS, Colbert AP. Magnetic and electromagnetic field therapy. J Back Musculoskel Rehabil 2000; 15(1):17-29.

24. Sheffet A, Cytryn AS, Louria DB. Applying electric and electromagnetic energy as adjuvant treatment for pressure ulcers: a critical review. Ostomy Wound Manage 2000; 46(2):28-33, 3640, 42-44.

25. Wolcott LE, Wheeler PC, Hardwicke HM, Rowley BA. Accelerated healing of skin ulcers by electrotherapy: preliminary clinical results. South Med J 1969; 62:795-801.

26. Cuddigan J, Frantz RA. Pressure ulcer research: pressure ulcer treatment. A monograph from the National Pressure Ulcer Advisory Panel. Adv Wound Care 1998; 11(6):294-300.

27. Markov MS, Pilla AA. Electromagnetic-field stimulation of soft-tissues-pulsed radio-frequency treatment of postoperative pain and edema. Wounds-A Compendium of Clinical Research and Practice 1995; 7(4):143-151.

28. Gardner SE, Frantz RA, Schmidt FL. Effect of electrical stimulation on chronic wound healing: a meta-analysis. Wound Repair Regen 1999; 7(6):495-503.

29. Akai M, Hayashi K. Effect of electrical stimulation on musculoskeletal systems; A meta-analysis of controlled clinical trials. Bioelectromagnetics 2002; 23(2):132-143.

30. Kloth LC, Feedar JA. Acceleration of wound-healing with high-voltage, monophasic, pulsed current. Phys Ther 1988; 68(4):503-508.

31. Feedar JA, Kloth LC, Gentzkow GD. Chronic dermal ulcer healing enhanced with monophasic pulsed electrical stimulation. Phys Ther 1991; 71(9):639-649.

32. Lundeberg TCM, Eriksson SV, Malm M. Electrical nerve stimulation improves healing of diabetic ulcers. Ann Plast Surg 1992; 29:328-331.

33. Jerčinović A, Karba R, Vodovnik L, Stefanovska A, Krošelj P, Turk R. Low frequency pulsed current and pressure ulcer healing. IEEE Trans Rehabil Eng 1994; 2(4):225-233.

34. Karba R, Šemrov D, Vodovnik L, Benko H, Šavrin R. DC electrical stimulation for chronic wound healing enhancement. Part 1. Clinical study and determination of electrical field distribution in the numerical wound model. Bioelectrochem Bioenerg 1997; 43:265-270.

35. Baker LL, Chambers R, DeMuth SK, Villar F. Effects of electrical stimulation on wound healing in patients with diabetic ulcers. Diabetes Care 1997; 20(3):405-412.

36. Barker AT, Jaffe LF, Vanable JW, Jr. The glabrous epidermis of cavies contains a powerful battery. Am J Physiol 1982; 242(3):358-366.

37. Robinson KR. The responses of cells to electrical fields - a review. J Cell Biol 1985; 101(6):20232027.

38. Sheridan DM, Isseroff RR, Nuccitelli R. Imposition of a physiologic DC electric field alters the migratory response of human keratinocytes on extracellular matrix molecules. J Invest Dermatol 1996; 106(4):642-646.

39. Nishimura KY, Isseroff RR, Nuccitelli R. Human keratinocytes migrate to the negative pole in 
direct current electric fields comparable to those measured in mammalian wounds. J Cell Sci 1996; 109:199-207.

40. Farboud B, Nuccitelli R, Schwab IR, Isseroff RR. DC electric fields induce rapid directional migration in cultured human corneal epithelial cells. Exp Eye Res 2000; 70(5):667-673.

41. Pullar CE, Isseroff RR, Nuccitelli R. Cyclic AMP-dependent protein kinase a plays a role in the directed migration of human keratinocytes in a DC electric field. Cell Motil Cytoskeleton 2001; 50(4):207-217.

42. Gault WR, Gatens PF. Use of the low intensity direct current in management of ischemic skin ulcers. Phys Ther 1976; 56:265-269.

43. Munih M, Ichie M. Current status and future prospects for upper and lower extremity motor system neuroprostheses. Neuromodulation 2001; 4(4):176-185.

44. Bajd T, Cikajlo I, Šavrin R, Erzin R, Gider F. FES rehabilitative systems for re-education of walking in incomplete spinal cord injured persons. Neuromodulation 2000; 3(3):167-174.

45. Likar B, Poredoš P, Prešern-Štrukelj M, Vodovnik L, Klešnik M. Effects of electric current on partial oxygen tension in skin surrounding wounds. Wounds-A Compendium of Clinical Research and Practice 1993; 5(1):32-36.

46. Feedar JA, Kloth LC. Acceleration of wound healing with high-voltage pulsating direct current. Phys Ther 1985; 65(5):741.

47. Mawson AR, Siddiqui FH, Connolly BJ, Sharp CJ, Stewart GW, Summer WR, et al. Effect of high-voltage pulsed galvanic stimulation on sacral transcutaneous oxygen-tension levels in the spinal-cord injured. Paraplegia 1993; 31(5):311-319.

48. Bassett CAL. Bioelectromagnetics in the service of medicine. Electromagnetic Fields 1995; 250:261-275.

49. Bassett CAL. Beneficial-effects of electromagnetic-fields. J Cell Biochem 1993; 51(4):387-393.

50. vanRijswijk L. Wound assessment and documentation. Wounds-A Compendium of Clinical Research and Practice 1996; 8(2):57-69.

51. Lazarus GS, Cooper DM, Knighton DR, Margolis DJ, Pecoraro RE, Rodeheaver G, et al. Definitions and guidelines for assessment of wounds and evaluation of healing. Arch Dermatol 1994; 130:489-493.

52. Maklebust JA. Pressure ulcer assessment. Clin Geriatr Med 1997; 13(3):455-481.

53. Plassmann P, Jones TD. MAVIS: a non-invasive instrument to measure area and volume of wounds. Med Eng Phys 1998; 20(5):332-338.

54. Krouskop TA, Baker R, Wilson MS. A noncontact wound measurement system. J Rehabil Res Dev 2002; 39(3):337-345.

55. Richard JL, Daures JP, Parer-Richard C, Vannereau D, Boulot I. Of mice and wounds reproducibility and accuracy of a novel planimetry program for measuring wound area. WoundsA Compendium of Clinical Research and Practice 2000; 12(6):148-154.

56. Patete PV, Bulgrin JP, Shabani MM, Smith DJ. A non-invasive, three-dimensional, diagnostic laser imaging system for accurate wound analysis. Physiol Meas 1996; 17(2):71-79.

57. Rajbhandari SM, Harris ND, Sutton M, Lockett C, Eaton S, Gadour M, et al. Digital imaging: an accurate and easy method of measuring foot ulcers. Diabet Med 1999; 16(4):339-342.

58. Marjanovic D, Dugdale RE, Vowden P, Vowden KR. Measurement of the volume of a leg ulcer using a laser scanner. Physiol Meas 1998; 19(4):535-543.

59. Covington JS, Griffin JW, Mendius RK, Tooms RE, Clifft JK. Measurement of pressure ulcer volumes using dental impression matherials: suggestions from the field. Phys Ther 1989; 69:690694.

60. Helbich TH, Roberts TPL, Rollins MD, Shames DM, Turetschek K, Hopf HW, et al. Noninvasive assessment of wound-healing angiogenesis with contrast-enhanced MRI. Acad Radiol 2002; 9:S145-S147.

61. Jones TD, Plassmann P. An active contour model for measuring the area of leg ulcers. IEEE Trans Med Imaging 2000; 19(12):1202-1210.

62. Stefanovska A, Vodovnik L, Benko H, Turk R. Treatment of chronic wounds by means of electric and electromagnetic fields. Part 2. Value of FES parameters for pressure sore treatment. Med Biol Eng Comput 1993; 31:213-220. 
63. Mayrovitz HN. Shape and area measurement considerations in the assessment of diabetic plantar ulcers. Wounds-A Compendium of Clinical Research and Practice 1997; 9(1):21-28.

64. Kantor J, Margolis DJ. Efficacy and prognostic value of simple wound measurements. Arch Dermatol 1998; 134(12):1571-1574.

65. Shea JD. Pressure sores-classification and management. Clin Orthop 1975; 112:89-100.

66. Russell L. Pressure ulcer classification: the systems and the pitfalls. Br J Nurs 2002; 11(12):4959.

67. Ferrell BA, Artinian BM, Sessing D. The sessing scale for assessment of pressure ulcer healing. J Am Geriatr Soc 1995; 43:37-40.

68. Bartolucci AA, Thomas DR. Using principal component analysis to describe wound status. Adv Wound Care 1997; 10(5):93-95.

69. Stotts NA, Rodeheaver GT, Thomas DR, Frantz RA, Bartolucci AA, Sussman C, et al. An instrument to measure healing in pressure ulcers: Development and validation of the Pressure Ulcer Scale for Healing (PUSH). Journals of Gerontology Series A-Biological Sciences and Medical Sciences 2001; 56(12):M795-M799.

70. Johnson M, Miller R. Measuring healing in leg ulcers: Practice considerations. Appl Nurs Res 1996; 9(4):204-208.

71. Gilman TH. Parameter for measurement of wound closure. Wounds 1990; 3:95-101.

72. Cukjati D, Reberšek S, Karba R, Miklavčič D. Modelling of chronic wound healing dynamics. Med Biol Eng Comput 2000; 38(3):339-347.

73. Cukjati D, Reberšek S, Miklavčič D. A reliable method of determining wound healing rate. Med Biol Eng Comput 2001; 39(2):263-271.

74. Johnson M. Using cluster analysis to develop a healing typology in vascular ulcers. J Vasc Nurs 1997; 15:45-49.

75. Lyman IR, Tenery JH, Basson RP. Corelation between decrease in bacterial load and rate of wound healing. Surg Gynecol Obstet 1970; 130(4):616-620.

76. Birke JA, Novick A, Patout CA, Coleman WC. Healing rates of plantar ulcers in leprosy and diabetes. Leprosy Rev 1992; 63:365-374.

77. Gorin DR, Cordts PR, LaMorte WW, Menzoian JO. The influence of wound geometry on the measurement of wound healing rates in clinical trials. J Vasc Surg 1996; 23:524-528.

78. Cuddigan J. Pressure ulcer classification: What do we have? What do we need? Adv Wound Care 1997; 10(5):13-15.

79. Karba R, Vodovnik L, Prešern-Štrukelj M, Klešnik M. Promoted healing of chronic wounds due to electrical stimulation. Wounds 1991; 3(1):16-23.

80. Skene AI, Smith JM, Doré CJ, Charlett A, Lewis JD. Venous leg ulcers: a prognostic index to predict time to healing. BMJ 1992; 305:1119-1121.

81. Kantor J, Margolis DJ. A multicentre study of percentage change in venous leg ulcer area as a prognostic index of healing at 24 weeks. Brit J Dermatol 2000; 142(5):960-964.

82. Robnik-Šikonja M, Kononenko I. An adaption of Relief for attribute estimation in regression. In: Dough F, ed. Machine Learning: Proceedings of the Fourteenth International conference on Machine learning. San Mateo, California: Morgan Kaufman Publ., 1997:296-304.

83. Cukjati D, Robnik-Šikonja M, Reberšek S, Kononenko I, Miklavčič D. Prognostic factors in the prediction of chronic wound healing by electrical stimulation. Med Biol Eng Comput 2001; 39(5):542-550.

84. Breiman L, Friedman JH, Olshen RA, Stone CJ. Classification and regression trees. Belmont, California: Wadsworth International Group, 1984.

85. Herrick SE, Sloan P, Mcgurk M, Freak L, Mccollum CN, Ferguson MWJ. Sequential changes in histologic pattern and extracellular-matrix deposition during the healing of chronic venous ulcers. Am J Pathol 1992; 141(5):1085-1095.

86. Reger SI, Hyodo A, Negami S, Kambic HE, Sahgal V. Experimental wound healing with electrical stimulation. Artif Organs 1999; 23(5):460-462.

87. Yenpatton GPA, Patton WF, Beer DM, Jacobson BS. Endothelial-cell response to pulsed electromagnetic-fields-stimulation of growth rate and angiogenesis in vitro. J Cell Physiol 1988; 134(1):37-46. 